Access to Opioid Use Disorder Treatment for Pregnant and Postpartum Women: Challenges, Barriers and Opportunities in Montana

JG Research \& Evaluation | Bozeman, MT 


\section{REPORT INFORMATION AND ACKNOWLEDGEMENTS}

This report was written by Kristal Jones, Brandn Green, and Tabitha Stickel of JG Research \& Evaluation and Katie Loveland of Loveland Consulting.

The authors would like to thank all of the respondents who took the time to be interviewed for this report. Thank you as well to Joclynn Ware (AMDD SOR grant manager), Bobbi Perkins (Prevention Bureau Chief), and Ki-Ai McBride (Opioid Prevention Program Manager).

This study was funded by the Montana State Opioid Response (SOR) grant from the Substance Abuse and Mental Health Services Administration (SAMHSA), U.S. Department of Health and Human Services (HHS) to the Addictive and Mental Disorders Division (AMDD), Montana Department of Public Health and Human Services (DPHHS).

The study was reviewed by Western IRB and found to be exempt under 45 CFR $\S$ 46.104(d)(2).

The views and opinions expressed in this report are those of the authors and do not reflect the official policy or position of any agency of the government of the State of Montana.

This report was finalized in August of 2021.

\section{CITATIONS OF THIS PAPER}

Please use the following format when citing this paper:

Jones, K., Green, B., Loveland, K. and Stickel, T. (2021). Access to Opioid Use Disorder Treatment for Pregnant and Postpartum Women: Challenges, Barriers and Opportunities in Montana. JG Research and Evaluation. DOI: 10.36855/SOR2021.2. Available at: jgresearch.org/ publications. 


\section{TABLE OF CONTENTS}

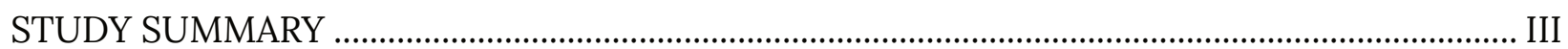

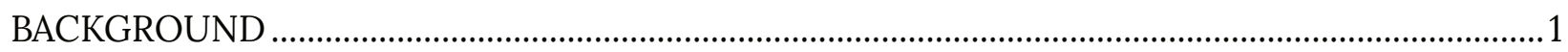

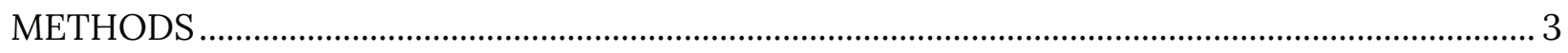

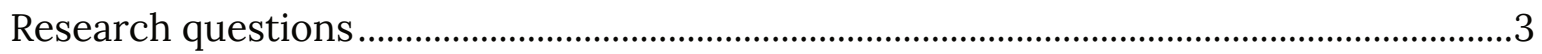

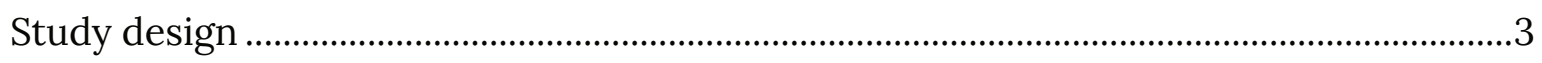

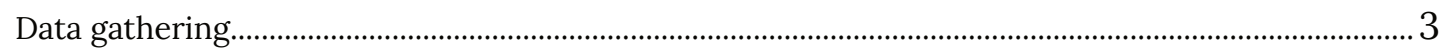

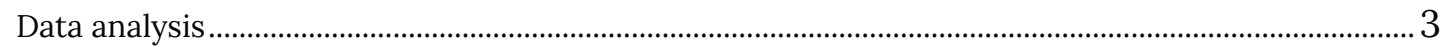

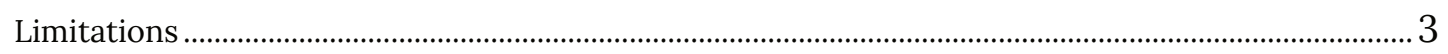

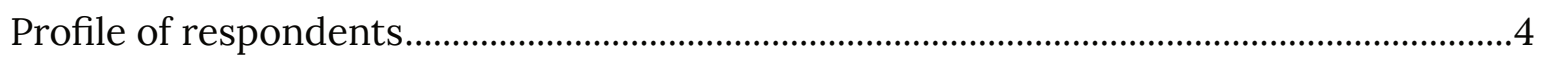

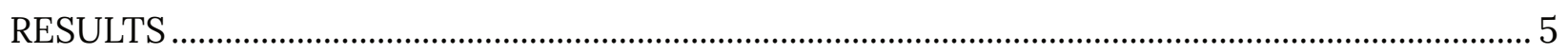

Characteristics of OUD among pregnant women............................................................

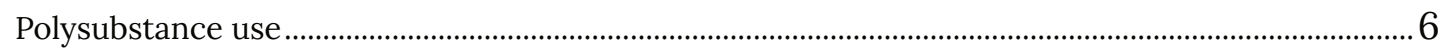

Co-occurring mental health conditions and trauma .......................................................................... 6

Community profiles of access to MOUD for pregnant women ............................................ 7

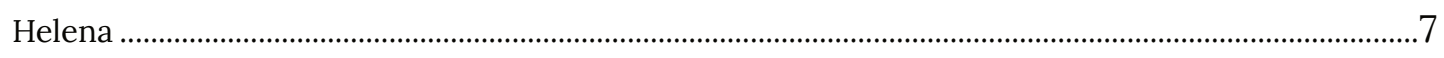

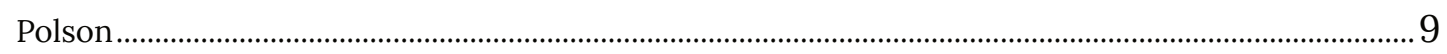

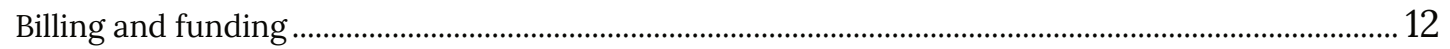

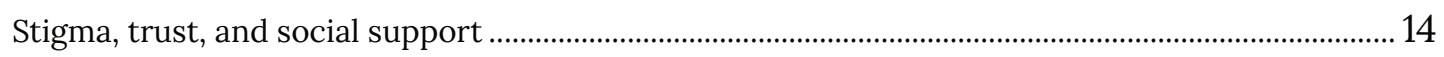

Gaps in the OUD treatment system for pregnant women ................................................18

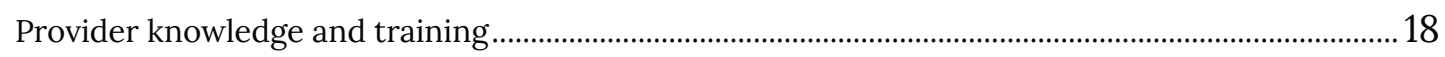

Ongoing treatment and recovery services ................................................................................................ 19

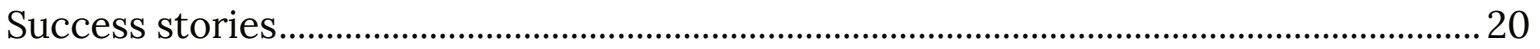

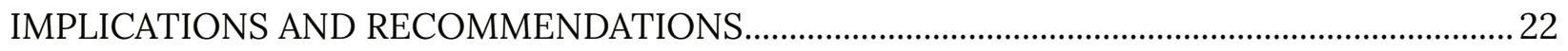

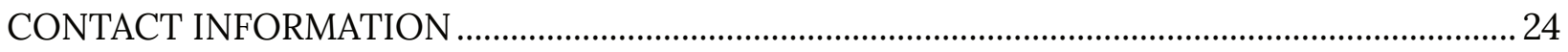




\section{STUDY SUMMARY}

Pregnant and postpartum women with opioid use disorder (OUD) are a priority population for federal and state efforts to address the opioid crisis. In the state of Montana, the absolute numbers of pregnant women with OUD are relatively small but the prevalence of neonatal abstinence syndrome (NAS) and newborns impacted by opioid use during pregnancy are above the national rate and have increased over the past few years. Increasing access to medication-assisted treatment for OUD for pregnant and postpartum women is therefore a goal of the State Targeted Response (STR) and State Opioid Response (SOR) grant programs within the state of Montana.

This study focuses on understanding the challenges and gaps for pregnant and postpartum women with OUD to access MOUD through interviews with medical providers, behavioral health care providers, and social service providers who provide care to pregnant women. The study focuses on two Montana communities, Polson and Helena, to describe the care systems in place for pregnant women to receive both prenatal and OUD care, with a focus on integration, coordination and awareness among providers and pregnant women.

The results of this study show that there are very effective efforts at providing integrated prenatal and behavioral health care through specific programs and among specific providers within the two study communities. However, identifying and engaging pregnant women with OUD in these efforts remains a challenge. MOUD providers note that most of their pregnant patients were already in treatment and then became pregnant, suggesting a need to focus on engaging women about OUD treatment more generally. Within the prenatal care setting, providers emphasized how rare it is for women to admit to OUD, due to a fear of their child being taken away and because of more general stigma associated with pregnancy and substance use.

Specific challenges to increasing access to MOUD for pregnant women include a lack of integration across prenatal medical care and OUD treatment services, limited billing options for long-term treatment, and substantial stigma from providers and pregnant women themselves. Gaps in the system include a lack of provider knowledge about local resources for OUD treatment and pregnancy and limited options for ongoing treatment and recovery in the postpartum period that are tailored to the experience of new mothers.

Taken together, the findings of this study suggest a need for increased education and public awareness of available OUD treatment resources for both providers and women, expanded investments in integrated approaches to providing both prenatal care and SUD treatment, and a shift away from a punitive approach to addressing substance use in pregnancy, which can exacerbate fear and stigma in ways that keep pregnant women from seeking any kind of care. 


\section{BACKGROUND}

As opioid use disorder (OUD) has increased across the United States over the past decade, federal agencies have worked to address impacts on underserved populations and individuals with unique prevention and treatment needs. One of these special populations is pregnant and postpartum women. Addressing OUD in this population is a priority because of the challenges of receiving substance use disorder (SUD) treatment alongside prenatal care and because of the potentially negative impacts of ongoing substance use during pregnancy on both mother and babies. The Substance Abuse and Mental Health Services Administration (SAMHSA) has highlighted the need for changes in legal and medical systems to support, rather than punish, pregnant women with OUD and has developed clinical guidelines for OUD treatment during pregnancy ${ }^{1}$. However, rates of neonatal abstinence syndrome (NAS) have continued to rise, from 3.4 per 1,000 hospital births in $2009^{2}$ to 7.3 per 1,000 hospital births in $2017^{3}$.

In the state of Montana, NAS rates have been slightly above the national average and as of 2019 stood at 8.4 per 1,000 hospital births (Figure 1). Although NAS can be the result of the misuse of many different substances, hospital discharge records suggest that opioids are increasingly noted as effecting a small minority of newborns (1.8 per 1,000 hospital births in 2019, which is triple the rate in $2018^{4}$ ).

\section{Figure 1. Neonatal abstinence syndrome rates in MT and the US}

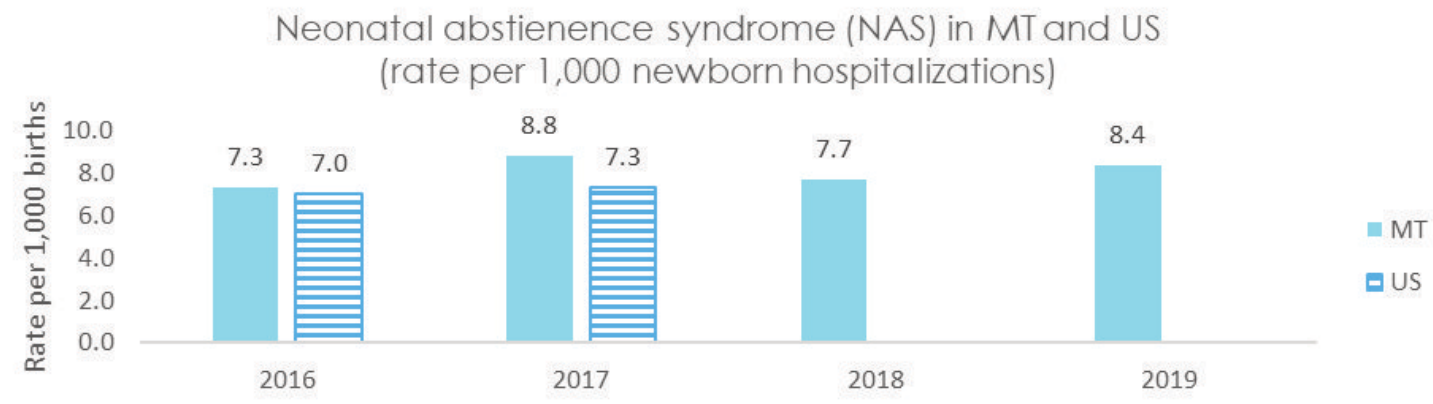

Source: MT Hospital Discharge Data System and Healthcare Cost and Utilization Project (HCUP) ${ }^{5}$

In addition to these clinical measures, best practices in integrated behavioral health and maternal health care include universal screening for behavioral health needs among pregnant women. In Montana, $82 \%$ of pregnant women reported that they were asked about illicit drug use during prenatal visits, which is good coverage but falls short of the universal screening goal (Figure 2). In contrast, virtually all pregnant women report being asked about alcohol, tobacco, and prescription drug use. In addition, MT PRAMS data suggests that 12\% of pregnant women in Montana do not receive prenatal care during the first trimester and

\footnotetext{
${ }^{1}$ Substance Abuse and Mental Health Services Administration. Clinical Guidance for Treating Pregnant and Parenting Women With Opioid Use Disorder and Their Infants. HHS Publication No. (SMA) 18-5054. Rockville, MD: Substance Abuse and Mental Health Services Administration, 2018.

${ }^{2}$ Ibid.

${ }^{3}$ Data from NAS Map, Healthcare Cost and Utilization Project (HCUP) (https://www.hcup-us.ahrq.gov/faststats / NASMap).

${ }^{4}$ Data from MT Hospital Discharge Data System (direct request made)

${ }^{5}$ Ibid. notes 3 and 4
} 
the Montana Office of Vital Statistics estimates that 25\% of pregnant women do not receive prenatal care in the first trimester ${ }^{6}$. This means that roughly $30-40 \%$ of pregnant women are not being screened for illicit drug use.

\section{Figure 2. Proportion of women reporting screening for pregnancy risk factors}

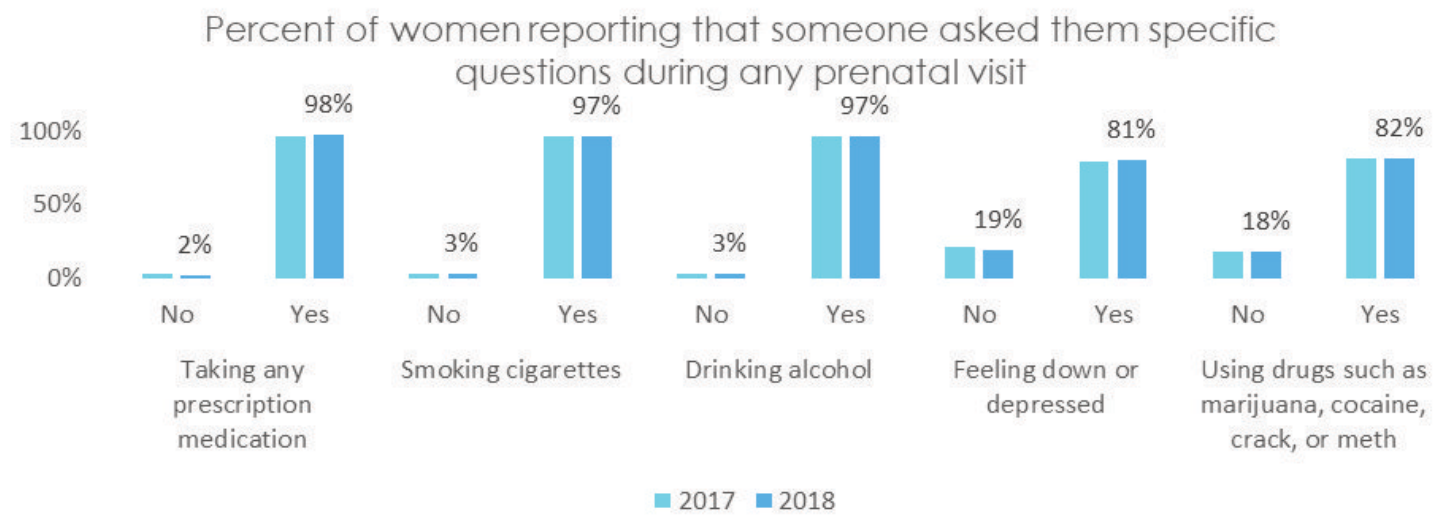

Source: $M T P R A M S^{7}$

In the context of ongoing need nationally, State Targeted Response (STR) and State Opioid Response (SOR) funds from SAMHSA included a focus on increasing access to OUD treatment and improving outcomes for pregnant and postpartum women. The State of Montana has supported treatment providers in developing programs specifically targeting pregnant and postpartum women over these funding cycles, and providers report small but consistent improvements in program access and participant outcomes. Further, overall numbers of pregnant women served specifically by medication for opioid use disorder (MOUD) programs remain low in Montana. Within the GPRA data collected by SOR grantees, 12 of the 685 clients served were pregnant women (under $2 \%$ of the total clients served).

This report is part of a series of studies on special populations as part of the State of Montana SOR grant program. The purpose is to better understand the current challenges and barriers to expanding access to OUD treatment, specifically MOUD, and improving outcomes for pregnant and postpartum women. Because pregnancy is both a unique and finite time, this report focuses on the specific relationship between prenatal care and MOUD, and the treatment and recovery needs of women in the postpartum and early childhood period. To characterize the current experiences of pregnant and postpartum women with OUD in Montana, we used a case study methodology to understand the screening, referral, treatment, and recovery systems in two Montana communities, Polson and Helena. Polson is a secondary population center in northwest Montana and is adjacent to the Flathead Indian Reservation. Helena is a primary population center in west-central Montana and is the state capital. All data are qualitative and are presented here as a summary of iterative thematic coding. The results demonstrate that there are many challenges related to lack of integration across medical and behavioral health care settings, as well as lack of knowledge and capacity from medical providers, recovery organizations, and pregnant women for OUD treatment.

\footnotetext{
${ }^{6}$ Data from the MT Office of Vital Statistics (http://ibis.mt.gov/query/result/birth/PNCCnty/PNCTri1.html)

${ }^{7}$ Data from MT Pregnancy Risk Assessment Monitoring System (PRAMS) (direct request made)
} 


\section{METHODS}

\section{Research questions}

1. What is the burden of OUD among pregnant women in MT?

2. When there are women with OUD, are local referral sources aware of and connecting them with the MOUD providers?

3. If referral sources are making connections to MOUD for pregnant women, what are the barriers to enrollment?

\section{Study design}

\section{Data gathering}

As one arm of a multi-faceted, qualitative study to understand treatment and recovery capacity for individuals with OUD in Montana, 26 interviews were conducted with individuals working at relevant medical and community support organizations across Lewis and Clark County (Helena) and Lake County (Polson). Interviews were conducted by one researcher, with other members of the project team providing logistical support and support for data analysis. The study used two case study communities as a method for understanding the dynamics pregnant women and mothers with SUD may experience as they attempt to navigate the local care system. These two communities were selected because they include SOR-funded MOUD sites, represent geographic diversity, and have different histories related to the development of programs intended to serve pregnant women with SUD/OUD. Data collection for the interviews followed a three-step process with an initial formal recruitment email and a follow-up call, a phone or conferencing call interview, typically lasting between 30 and 60 minutes and audio recorded (with permission), and a request for recommendations for other individuals to interview (snowball sampling). Interviews with providers and staff included questions about their experiences working with pregnant women in the MOUD program and other providers who serve them. In addition, the broader evaluation project of which this report is a part has interviewed currently active MOUD clients over the past two years. A small number of these were recently pregnant and either using opioids or receiving MOUD, and we include quotes from these interviews throughout this report as well.

\section{Data analysis}

Audio recordings were transcribed and analyzed with NVivo software using a coding scheme developed by the research team. Audio recordings of all interviews were downloaded from shared online storage and transcribed using a third-party service, Rev. All transcripts were uploaded into NVivo software and shared through a collaborative cloud system. Data analysis had four phases: creation of coding schema by the study leads with collaborative input from the research team; an initial round of coding (three transcripts) by an assigned analyst; review of initial coding of three assigned transcripts with study leads and the analyst for quality assurance across the study's transcripts; and, finally, detailed coding of all remaining transcripts.

\section{Limitations}

The interviews conducted represent a cross-section of individuals who work within the healthcare system and offer key services for pregnant women and mothers with SUD/OUD 
in two communities, Helena and Polson. Noticeably missing, though, are interviews with pregnant women and mothers themselves. During the two stages of recruitment-the initial round of recruitment done with email and phone calls, and the recruitment for other,

potential interviewees at the end of each interview-the researcher asked for referrals to any pregnant women or mothers with OUD who might be willing to participate in the study. Ultimately, no women elected to participate. We do include perspectives from very few current MOUD clients who have recently been pregnant, but the conclusions drawn in this report are from the perspective of those working within and around the healthcare system, rather than those that are patients of that system. Interviews were only conducted in two main locations. Though it is not unreasonable to suspect these communities represent similar healthcare landscapes found throughout Montana, other locations may have additional, and specialized needs, not discussed by those residing in Polson and Helena. A final limitation was the lack of IRB approval from the Confederated Salish and Kootenai Tribe, which mean that no Tribal members working within tribal lands could be interviewed. However, we did speak with providers who work with populations in Lake County that include Tribal members.

\section{Profile of respondents}

This report is based on the interviews done with 26 different individuals working with at-risk pregnant women and mothers in Helena or Polson. Individuals interviewed worked at a variety of organizations including hospitals and other healthcare facilities, Child and Family Services Division (CFSD), and non-profits. Interviewees varied in the length of time spent in the field, ranging from a few years to a few decades. Additionally, interviews were done with people holding different titles, including obstetricians (OBs) and primary care physicians; nurses; mental health counselors; non-profit directors and workers; CFSD staff; and SUDspecific providers. In addition, all SOR-funded sites focus on pregnant women as a priority population.

\begin{tabular}{lc}
\multicolumn{1}{c}{ Respondent type } & Number of respondents \\
\hline Helena & \\
\hline Behavioral health provider & 4 \\
\hline Maternal health provider & 2 \\
\hline Public health and social service provider & 4 \\
\hline Nonprofit service provider & 3 \\
\hline Polson & 2 \\
\hline Behavioral health provider & 6 \\
\hline Maternal health provider & 1 \\
\hline Public health and social service provider & NA
\end{tabular}




\section{RESULTS}

\section{Characteristics of OUD among pregnant women}

The relationship between OUD treatment and pregnancy is important in terms of understanding the most common and impactful referral pathways and points of integration in the continuum of care. A consistent observation by both OB/GYN staff and MOUD providers is that OUD treatment almost always precedes pregnancy for their clients.

I've never had anybody admit to the opioids. And even when I worked at the OB's [office], some of the moms that we came in, a lot of times they were late getting prenatal care, they came in late...Most of them were already in [OUD] treatment by the time they came. So, I almost wonder if they got treatment because of being in... They got prenatal care due to the fact that they were already being treated [for OUD]. (Helena maternal health provider)

Quite a few of our pregnant patients were with us and then, you know...everything came together, lives are going well and they decided they want to get pregnant. (Polson behavioral health provider)

Providers did note that they consistently see pregnant women with OUD who are illicitly using certain MOUD prescriptions (e.g., Subutex and Sublocade) as opposed to through treatment providers. This use is intended to manage their OUD during pregnancy without having to engage a treatment provider.

I've had several families and moms who later told me they were using Suboxone illegally, illicitly but trying to do it in a responsible way...I think, I honestly think people have the best of intentions. It's not often you want to be pregnant when you're 19 and addicted to heroin. I really think people are trying to do the right thing. But some of the options are limited. (Polson behavioral health provider)

A lot of them are not getting treatment and [instead] getting treatment off the streets. So I've had a couple manage themselves through pregnancy because they've been able to come up with medications, and then they came to me at the end of pregnancy, informed me of what they were doing... When they came to prenatal visit at 36 weeks, and I hadn't seen them the whole pregnancy. So, a lot of them are, like I said, with the guilt situation, it's makes a big barrier to seeking health care. (Polson maternal health provider)

When asked to estimate the prevalence or burden of OUD for pregnant women, providers in Helena tended to see opioid use during pregnancy as uncommon, whereas in Polson it is commonly seen. Many providers, whether they are OB/GYNs, primary care providers, mental health providers, or social service providers, do not consistently conduct urine screening for substances. Instead, they rely on self-reporting by women seen during pregnancy or on observations while women are in the hospital during the delivery.

I just document substances that were either reported that the mother was using, like self-reported by the mother during pregnancy. Or what they were positive for at the time of delivery. Which I think is a gross under representation of the epidemic at hand. (Helena maternal health provider)

A common refrain from interviewees was that pregnant women who use opioids, or any other illegal or controlled substance, are not doing so in a vacuum. For many pregnant 
women and mothers, their substance use is occurring alongside significant others, family members, and friend groups that also use heavily. As one MOUD provider explained, familial use is often a barrier to sobriety and recovery as "all the family members have continued to use, and that was often the trigger, often a factor related to relapse." For many pregnant women, maintaining recovery became impossible when, as one provider noted, "it's sitting on their kitchen table at the house."

Providers also spoke of several commonalities in their clients with OUD, including polysubstance use, co-occurring mental health disorders, and past experiences of trauma. As one person with CFSD observed: "generally we're dealing with people who already have substance abuse problems, or they have mental health disorders. Or they are chronically homeless or living in poverty."

\section{Polysubstance use}

Interview respondents reported high rates of marijuana, methamphetamine, and alcohol use alongside opioid use among pregnant women.

Pretty routinely, [we] see substance abuse, a lot of marijuana use during pregnancy, which we're trying to avoid. Heroin is very common and other opiates like prescription opiate use. And at one point last fall, I had like three or four in a week that I was trying to get onto buprenorphine or dealing in some way with withdrawal, infant withdrawals, or trying to get mom onto something to help deal with the heroin withdrawals during pregnancy. (Polson maternal health provider)

I would say alcohol use disorder is almost a baseline for pretty much anybody that we serve. Sometimes it's the main drug of choice. I would say ...Methamphetamine is probably the number one, with opiates in the form of both pain medication and heroin being a close second, and sometimes those two, of course, are used together. So that there's methamphetamine use and then the opiates are used to come down, as well as the alcohol. (Helena nonprofit)

So we've been running statistics on substance use in our hospitals since 2013. And sadly our percentage of moms or babies, or both, who test positive for substances is always right around 22 or $25 \%$, somewhere between 20 and $25 \%$. And that average hasn't been affected by...[an] IMAT [integrated medicationassisted treatment, now called MOUD] program now that's pretty robust for Subutex for people that have opioid addiction in those substances. (Polson maternal health provider)

\section{Co-occurring mental health conditions and trauma}

Behavioral health treatment providers who work with pregnant women emphasized that those with SUD almost always have co-occurring mental health conditions.

I cannot think of one pregnant woman I worked with that didn't have a cooccurring diagnosis, so a lot of major depressive disorder. I think every single one was probably, if I could look back in their records, probably qualified as also having post-traumatic stress disorder... but also generalized anxiety, a lot of attachment concerns, so a lot of women that had a history of being removed from family members or in some sort of different caregiving role as a child, and maybe didn't have a consistent family member or caregiver present in their life. (Polson behavioral health provider) 
Providers mentioned a variety of mental health concerns pregnant patients face, including depression, both during and after pregnancy, and trauma. Addressing SUD and co-occurring mental health disorders for pregnant women requires tailored approaches that fit this particular period of life. For some providers, this means prioritizing short-term stability over recovery; one nurse noted that her focus was often "just trying to help them keep that stable through the pregnancy and then to the postpartum period." For others, this means taking a treatment approach that directly engages with a woman's identity as a mother or mother-tobe. However, as one mental health provider stated, "trying to find modalities that fit, trying to find supports that fit, that have the lens of maternal mental health has been a bit of a struggle."

Many interview respondents highlighted that among pregnant women with SUD in general, trauma is a consistent and challenging co-occurring mental health condition that ideally needs to be addressed through integrated treatment.

I think that when we're working with pregnant women, I know that the topic here is kind of substance use or opioid use, but we have to recognize that it's often related to early childhood trauma. And we need to start having a discussion on how treatment supports... Like you said, it's been tabled for so long. In our world it's kind of been like, what comes first? The chicken or the egg? Is it substance abuse [treatment] or is it therapy? And there is no answer to that question. (Helena behavioral health provider)

Some providers specified their clients had complex trauma histories from childhood, while others described clients with entire lifetimes of traumatic experiences.

The trauma history of our girls is hard. They come in with a variety of experiences, but the trauma severity goes pretty deep. We've had girls who've been trafficked since they were seven, eight years old, who've undergone sexual trauma, emotional abuse, physical abuse, neglect. If you can think of all the horrible things that happen to an individual, most of them have experienced a lot of it. (Helena behavioral health provider)

Experiences of trauma create an additional barrier to establishing the types of relationships needed between treatment providers and clients to address any type of OUD.

... One of the things that's just so clear is the trauma that these women have. And not just women. I have a whole practice of people who have been traumatized, who have grown up in just horrendously traumatic environments of all kinds of abuse and neglect. And so it's like, why would you trust me? That's really something that I think they face. (Helena behavioral health provider)

\section{Community profiles of access to MOUD for pregnant women}

\section{Helena}

Helena is somewhat of a regional hub for medical care and other social service providers, and some of the best-known SUD treatment providers for pregnant women and mothers in the state are based there, including Boyd Andrew, Florence Crittenton, and the YWCA. Helena is also one of the largest Montana cities and has several MOUD providers. The most highprofile MOUD providers in Helena are Helena Indian Alliance (often referred to as 'Leo Pocha') and PureView Health Center. Interview respondents highlighted that both organizations provide integrated MOUD and other SUD treatment, which is helpful for clients with polysubstance use and co-occurring mental health disorders. However, neither 
provide prenatal or OB/GYN care, and thus receiving MOUD care while pregnant requires engaging with at least two facilities that have few formal organizational connections. There are some opportunities to integrate prenatal care, both physical and behavioral, with MOUD. For example, the staff at Florence Crittenton stated that the medical director is a waivered provider, but that they have not utilized MOUD for treating OUD. Staff at St. Peter's noted that the health system was planning to hire a waivered provider in fall $2020^{8}$.

Behavioral health, physical health, and social service providers across the spectrum in Helena talked about how the continuum of care for SUD treatment is in general fairly integrated aside from the inclusion of MOUD, particularly for pregnant patients. Family Treatment Court was described by many providers as a hub for referring patients to other treatment and recovery services. As one CFSD staff member explained, "If they are involved with treatment court, they can get into places like the YWCA more quickly... it's a lot higher level of services available through the Family Treatment Court than a typical CPS case." Similarly, a YWCA staff member noted that "probably 95\% of the pregnant women we've served currently and in the past are involved in the criminal justice system in some way. They tend to be also involved with Child and Family Services."

Florence Crittenton, a facility in Helena for pregnant and parenting teens and young adults, has recently become a state approved substance use treatment provider and is working to integrate care.

We are really working hard to try to create those partnerships and build that continuum of care. ...we're really trying to do is build our own system that allows these women to continue in outpatient with the same clinicians that they had with us, maybe when they were in residential, or at the very least on the same clinical team. There's such a trust that's built during that continuum... We've also on the other end of it really been working hard to try to build some of those partnerships. Like I said, we're trying to work with, have an MOU [memorandum of understanding] with Helena OBGYN through their Meadowlark Initiative. We have an MOU with options in women's clinic, had lots of conversations with the Friendship Center, trying to build that same continuum of care and have lots of entry points for women to be able to access these services. (Florence Crittenton staff member)

At St. Peter's hospital, integrated behavioral healthcare for pregnant women is also clearly established through participation in the Meadowlark Initiative $^{9}$ and other ongoing investments.
So, St. Peter's, we have an integrated model that we've been doing for the last three years now. Well, maybe two. We started off in our position as nurses and quickly realized that, "Oh my goodness... We can't talk about their physical health without addressing mental health and social situations first or right along with it." That's kind of how we work is right along with it... We have an integrated team of five behavioral health professionals, which are therapists. I think that we have seven nurses now, two social workers and two pharmacists. We all work together on patients and all of us, we either collaborate [or] even jointly meet with patients. But at times, just say, "Hey, do you have ideas for this situation?" That type of a thing too. (Helena maternal health provider)

\footnotetext{
${ }^{8}$ Dr. Kyle Moore joined St. Peter's Health Medical Group in August 2020: https://www.sphealth.org/storiesnews/news/st-peters-health-medical-group-welcomes-family-medicine-physician-and-addiction.

${ }^{9}$ The Meadowlark Initiative is funded by the Montana Health Care Foundation: https://mthcf.org/themeadowlark-initiative/.
} 
Despite the existence of diverse resources in Helena, behavioral health providers expressed a general lack of awareness about exactly if, or how, pregnant women with OUD who are seeking MOUD might be served in a more integrated way.

I want to believe that they're receiving treatment elsewhere, whether it's through an OB/GYN clinic or some service in that form, but it's not widely discussed, if that makes sense, or promoted, that there's that particular facility that's focused on opioid addiction with those that are pregnant. (Helena behavioral health provider)

I think it's certainly the Meadowlark Initiative is growing that, having that integration of behavioral and physical health within the pregnancy care setting. And then requiring that connection to MAT or having that within the services. (Helena nonprofit)

Other medical and social service providers noted that even if they have a general awareness of MOUD programs, they are not always knowledgeable about the specific details and how the programs might work (or not work) for pregnant women.

There are kind of two little clinics that did medication-assisted treatment in Helena, but then I believe both of them are no longer functioning either. I think people, we just kind of didn't know enough about their system and what they were doing ...Then Leo Pocha [Helena Indian Alliance], I think that's also a great place to connect with... We just kind of haven't crossed paths I guess with it. Not that we're avoiding a connection, a relationship, but just kind of also not knowing all of what they offer either. (Helena nurse)

I guess what we get trained on is that the new workers, they go through our training program and they're trained about drug identification and treatment and stuff, but generally we don't deal much with the actual treatment process. We actually just refer out to the licensed addition counselors in town and the providers to make the recommendations for that, and however treatment looks, we just support them going to treatment. So they'll go get a chemical dependency evaluation that says you need intensive outpatient. We'll say, "Well, you can go to IOP [intensive outpatient]." That's generally what we do. We don't have the nuts and bolts of actually what occurs. I mean, everybody's got knowledge if they go to chemical dependency counseling, they talk about their issues with chemicals with the [chemical dependence] counselor. But we don't really know the nuts and bolts of what actually is represented past that. (Helena social service provider)

\section{Polson}

Polson is a small community that is also the hub of Lake County, and the most visible MOUD providers in the county are located at the Providence St. Joseph Medical Center ('St. Joseph's' or 'St. Joe's'). St. Joseph's is a good model for integrated care for pregnant women with OUD because of the possibility of receiving prenatal care, MOUD, and hospital delivery care in one location. St Joseph's creates an integrated continuum of care for pregnant women who receive prenatal services and are open to seeking treatment for OUD, and for women receiving MOUD to receive prenatal services if they become pregnant. One key to the success of this MOUD program and integration with other providers seems to be the MOUD providers themselves, and the trust they have created with patients through their approach to working with pregnant women. 
I actually think treatment, the stigma for treatment has changed probably in the last three to five years. I would say honestly I do think the IMAT program in Polson specifically has done a really nice job...I think [the provider's] patients really see her as a very trusted entity. I've had families go through pretty significant relapse, lose their children through court cases or custody and get, kind of fall off the bandwagon a bit if you will with use patterns. And feel really ashamed and ultimately on their own go and seek her out. And she just really offers a non-judgmental perspective. (Polson behavioral health provider)

Medical and behavioral health care providers not situated within the St. Joseph medical system noted that the St. Joseph MOUD providers are well-integrated into the broader continuum of care for pregnant women in Polson and across the county. This integration is positive for those providers who want to refer pregnant and post-natal clients to MOUD.

The program here at St. Joe's they do really well with our participants. They monitor them consistently. They're very open. They check on them. They check in with us there. They have a really good program up there for them. (Polson behavioral health provider)

Although the MOUD providers noted that most of their pregnant clients were already in treatment before getting pregnant, the results of prenatal and post-delivery screenings do refer some pregnant clients into their programs. Awareness of the St. Joseph approach to screening and offering integrated treatment options for OUD seems to have created a bit of a bifurcated care system within Lake County.

I think the other piece is we have a real fractionated obstetrical care here. There are family medicine doctors and $\mathrm{OB}$ providers who are doing things differently...So the hospital that I used to work at, St. Joe's, had a very firm protocol of watching for withdrawal. So consistent with the guidelines, if your baby was exposed to Suboxone, prescribed or unprescribed, they can withdraw and the recommendations are to monitor those babies for five days. And so, I think it kind of became a little bit of this cat and mouse game because different hospitals were doing things differently. A neighboring critical access hospital, St. Luke's, some people were trying to deliver there, so their baby wouldn't be monitored for withdrawal. And they wouldn't be aware, the records, there's not consistent records all the time of who's getting treatment. (Polson maternal health provider)

Unfortunately, we kind of have developed a little bit of a, not, not a reputation, but like St. Joe's is right down the street from St. Luke's [the community hospital]. And there is like the rumor mill says that, you know, when you go to St. Joe's... we do more stringent testing. And we test more for substances compared to St. Luke's, we manage differently... we keep the babies for a week and those types of things and St. Luke's doesn't do that... they'll send the moms home before baby even goes into withdrawal. And so, there's just some, some issues there. And so I think a lot of those moms that struggle with addiction kind of hear that through the grapevine. So, then they choose to go to St. Luke's. So oftentimes most of our patients, we end up seeing after they deliver and they decide that they want to get on the, the Suboxone. (Polson behavioral health provider)

Options for Tribal members are also somewhat bifurcated, with some access to MOUD, but also consistent barriers due to stigma, privacy concerns, and other challenges to accessing care. 
The tribe offers [MOUD] for tribal patients, but they put it on hold I think for a while because of staffing concerns. Tribal patients, they don't get their Suboxone covered outside of the tribe, so they would have to go through the tribal program to get it. That wasn't even an option for a while. That was a limitation. That is back up and running as far as I know, but there's a lot of barriers I think going through the tribe. Some privacy issues that people are worried about. (Polson maternal health provider)

I have heard many elders view opioid use and abuse as a white person's conspiracy to some degree. I mean those would be my words, but that this isn't a natural part of their community or culture. It was brought in as an outside influence, that it was prescribed and encouraged. And then it resulted in a significant problem and is very counter cultural. And now that there is a real severe problem, no one's helping.... And that the interventions that are being trialed are experimental and they're using a vulnerable population to get people addicted to something else. What else?...But in the native culture kids are revered and honored and I think you're seeing the effects here in the school system and in the family structure, of how challenging some behaviors are for kids who've been exposed, especially in utero to substances. (Polson behavioral health provider)

The integrated behavioral health approach taken by the Meadowlark Initiative sites and some MOUD sites that serve pregnant women is predicated on a comprehensive set of services being offered by the same organization, preferably at the same physical location. However, there is also a need to build connections across providers, offices, and organizations, to ensure that some of the benefits of integrated care can be conferred to individuals served by other providers.

I think this idea of continuity of care is so important and it's also so difficult because providers are so busy that it's really, really hard, it's really difficult to continue the communication, really good solid communication. I think it becomes really easy for clients to fall through the cracks, or to get frustrated or to not feel heard, to feel disregarded. I don't know what the answer is to that, besides perhaps more providers because when I think of all the mental health care providers that we have in our town, almost everybody has a waiting list. Unless you are set up like PureView or at Leo Pocha where you've got everything under one roof, I do think the communication gap is great. (Helena nonprofit)

As described in the community profiles of Helena and Polson, there is some integration across certain providers and institutions within the entire behavioral and prenatal care system, but there are many providers and institutions that are not a consistent part of this integration. In Polson, this was described as a 'factionated' prenatal care system, with a tightly integrated set of services built around the MOUD program for pregnant and postpartum women and a lack of engagement with that program by many providers and pregnant women. In Helena, it was noted that the MOUD providers are building integrated programs, including many types of physical and behavioral health services, but not OB/GYN care. In neither community did providers talk about integrating with other service providers outside of the medical and behavioral health treatment system. For example, models exist in other places that align treatment opportunities with Child Protective Services (CPS) processes to minimize the disruption caused by both treatment and intervention from CPS.

I think our problem in Montana is that we're a pretty rural state and we have limited resources...I've had workers that come from out of state, were CPS workers there, and they come here. It's like, "Well, we have a parent using heroin 
[where I moved from], we would take them to treatment the same day we remove their kids, and they would go to treatment for 30 days to get their kids back." Well, we don't have that here obviously. Right? So they would literally get into treatment the same day. So we don't have that one stop thing going on. They were attempting this a couple of years ago. They were talking about rolling out these addiction recovery team things. And the CPS...I know a couple of offices, they were getting that or they had that, which was a LAC [licensed addiction counselor] was going to be working in-house with the people, like LAC would be employed by a different agency, would have an office in the CPS office though, and would be able to offer immediate assistance to people who had the substance use disorder problems like with maintaining sobriety, that kind of stuff. (Helena social service provider)

The lack of full integration across types of providers and types of care for pregnant women with OUD highlights the need to build more robust referral mechanisms and relationships among providers. Interview respondents noted the possibility of building referral networks with other types of providers who interface with pregnant women and supporting those providers in referring clients beyond just the criminal justice system. Key to this type of referral network would be clear educational information and messaging that points to the opportunities for support, rather than punitive measures.

Right, right. Can we embrace this woman and say, "Okay, so here are some options. This is the education, this is what's going to happen to your baby if you continue using substances." There's the education piece. Not blaming. This is the biological result or potential biological result of substance use during pregnancy. Just education, not chastising. Then here the some options in the community, and not here's A, B, and C, you call them, but how about a warm handoff so that somebody else is helping to lead this woman in a way, again, that's non-judgmental, that is informative, that is dignified and respectful. I think we miss on this. We say, "You really ought to quit using, and if you don't, I'm going to call the powers that be." I'd love to see more of a community warm handoff. (Helena nonprofit)

But I wish that there were more resources that professionals could put these hands of these pregnant moms to let them know that you're not going to get in trouble. If you get clean right now, and if you stay clean that this could be a super-positive thing in your life. (Helena nonprofit)

\section{Billing and funding}

As with all SUD treatment services in the state of Montana, adequate billing options continue to be a challenge from the point of view of many providers. At the same time, there have been recent improvements and new opportunities, especially for billing Medicaid, which may have a positive impact for providers and clients. While none of these are unique to OUD, there are elements of billing for SUD treatment within an integrated care model that are especially relevant for pregnant women and are worth highlighting. For example, there are some general ways that medical billing limits integrated and holistic care, which are especially limiting when dealing with pregnant women with SUD.

If you're really looking at the population in a more narrow way of pregnant moms who are struggling with substance use disorder, I think it's really billing. I think you have to pay someone to do that nonbillable work in a big way. And I think you have to pay positions for the extra time it takes to have challenging conversations. I mean, I'm paid per patient I see. So I'm obviously not doing my 
job for the money, you can probably tell how I talk. But these are sensitive conversations that deserve some time and some thought and some care. These are not five-minute conversations that I just meet someone and say, 'Hey, this is how to not have your kid taken away,' right? (Polson maternal health provider)

As a clinician and as a social worker I feel like it would be my job [to help women find OUD treatment services], but there's no sustainability in that. I can't bill case management services, A, if she doesn't have Medicaid and B, because I'm a licensed clinician. The laws prohibit me to bill case management if it's not $51 \%$ of my job. But then who is responsible for helping this mom connect to the resources so that she can get the help that she needs? Therein lies one of our biggest gaps in this community. (Helena behavioral health provider)

In addition to the big-picture challenges of the dominant medical billing model for integrated care, providers consistently identified a few specific aspects of reimbursement rates that limit their ability adequately care for clients.

[There was a change in billing] the [treatment] group rate from an hourly rate per person to a per person-event. So if you have a two hour group, it used to be you could bill for say $\$ 20$ per hour... [now you can also bill for] certain core services. If you hit $\mathrm{X}$ number of core services in a week with a client, you get $\mathrm{X}$ number of dollars per day, which is a lot better than $\$ 17$ per event, but it's still, when you take a look at that in comparison to the hourly reimbursement rate for group, pales in comparison...And so you have programs that are fighting to just keep what they have. But it's always been underfunded. So there's not a narrative of how do we increase dollars to these programs. The programs are in a protection mode to not lose more. So it's not a growth type field. And that is very difficult...we have funding in a number of different pots, [Department of] Corrections money, DPHHS money. We've had stuff through the General Fund of the legislature, so we're more diversified than the average program in Montana, so we can probably weather more storms than a lot of programs can, but it's still really difficult to do good work when it feels like you're constantly getting squeezed in a vice with regard to the funding. (Helena behavioral health provider)

Not all billing challenges are related to Medicaid reimbursement rates and structures. For example, a Polson RN noted that "a lot of private insurance doesn't cover urine drug screens very well," making it more difficult to provide consistent accountability throughout the SUD treatment process. However, as noted by a Helena treatment provider, "this is kind of a great thing. Medicaid will now cover somebody who has actually been diagnosed with a substance use disorder, they can cover urine analysis, which is a huge part of support when you are trying to recovery is to be able to consistent UA's for accountability. That's a perk." Other providers specifically noted there is strong support for women with children to remain in long-term SUD treatment situations, and the positive impact that continued authorizations for treatment have on clients.

The idea of 30 and 60 day treatment is really... we're definitely finding that that's just not enough time and their ability to stay in recovery and continue to engage in services, and really make a big impact on their substance use, has to do with the amount of time that they are allowed to engage in program...I think that's something really important that we can't lose as a state. From a recovery home perspective, the state's been great. We have not had any continued say authorization issues. And I think that it really makes a difference. I look at some 
of our clients that were even doing well at three months, but if they were discharged at that point, I think we would know that they were going to struggle with that short amount of time. And we're looking at closer to 12 and 13 months stays. And when you can have your children with you, I think what was said about family-based treatment and when you can have your children, you're willing to put that time and you can be patient, even if you are maybe separated from your family members, it makes it a little bit easier pill to swallow to have to stay longer. (Helena behavioral health provider)

Overall, providers noted that there are some specific billing options, as well as block grants and similar funding opportunities, to support pregnant and postpartum women in receiving many kinds of SUD treatment. The SOR funding, with its focus on pregnant women as a priority population, as well as Meadowlark and other IBH investments, build on these otherfunding streams in ways that have created a relatively robust billing and funding environment for pregnant women seeking OUD treatment. At the same time, more general fiscal challenges faced by many SUD treatment providers are continually identified as a barrier to providing an adequate standard of care for all clients.

\section{Stigma, trust, and social support}

Issues of stigma have been consistently documented in the context of SUD treatment and recovery, with an important distinction between the impacts of received stigma (stigma from someone else toward the person with SUD) and self-stigma (stigma directed at oneself). Providers who work with pregnant women with OUD in Montana pointed to both types of stigma as leading to consistent challenges to improve treatment outcomes. Received stigma can come from medical providers (especially those focused on prenatal care, rather than behavioral health care), from social services providers or from the community at large. These stigmas create barriers to accessing treatment by making women nervous to disclose their OUD or seek treatment and undermining trust in service providers. On the other hand, selfstigma can create an additional emotional burdens for women with OUD, which adds a challenge to effective treatment and recovery.

In the most immediate sense, real and perceived stigma received from medical providers can impact the quality of medical care received by pregnant women with OUD, especially in relation to pain medication, and for prenatal and postpartum care. A few providers talked about the stigma they see from their colleagues toward women with OUD and the way that can create 'divergent care.'

You can say all you want that it's going to be non-judgmental experience and we're here to help. But the truth is if you deliver and if people don't think you're using substances, your delivery experience is different than if you told someone who has an addiction problem...And how the expectations are that you would interact with your baby and what opportunities you're given and how well you're listened to and how much you can advocate for yourself. How much pain medication you get. I think it's not stigma in that regard. And sometimes I get frustrated when people call it that, it's actually divergence in care. So you're signing up for sub-optimal care sometimes by admitting this is part of your health history. And that's unfortunate and I look at it different, but that's not stigma, that's racism. (Polson behavioral health provider)

I can think of a handful of conversations where [medical providers] very casually told me about using implicit bias in order to figure out who they do and don't screen. And very casually without a feeling of I need to be secretive about this. Just like no, this is kind of how we do it, blah, blah...So I don't like to talk in 
stereotypes, but it works pretty well here. You test a very young native American mom with holes in her jeans, but you don't test the mom with upper middle class, soccer mom, third kid. Or not test, excuse me. I should have used the word screen. But you don't screen that mom. (Helena nonprofit)

The fact that pregnancy is outwardly noticeable, especially in the third trimester, may decrease the likelihood that a woman with OUD will seek prenatal care or OUD treatment services if she is worried about received stigma. A staff member from CFSD in Helena noted that "we have people also who are trying to hide the fact that they're pregnant or they're not getting prenatal care. So, often times, we have gals who go to the hospital, who've had zero prenatal care the entire time. They don't want to get busted." As noted, the lack of integrated behavioral health settings that include prenatal care creates a logistical challenge for pregnant women and can also make concerns about stigma associated with a woman's pregnant appearance a challenge to accessing MOUD.

The stand-alone MAT clinics or specific doctor's offices or something, when you walk in there as a pregnant person, people know that you're a pregnant person. And that's a different level of stigma and shame to refer somebody out to an independent clinic that clearly is walking in there pregnant. So it doesn't surprise me at stand-alone or places where it's specific MAT and substance use treatment, that pregnant people don't want to show up there. (Helena nonprofit)

Self-stigma from pregnant women can also limit their seeking prenatal care and OUD treatment during the prenatal period, which can have long-term consequences for their children. At the same time, many providers, and the few women with OUD who have children with whom we spoke noted that many women balance this self-stigma and fear of received stigma of seeking OUD treatment with a desire to manage their OUD in a way that minimizes harm to the fetus.

I even struggle with it now today, even after being clean for over a year and a half. The stigma is just you're a horrible person and you don't care, and you don't care about your kid, and that it is by choice. Everybody, if they haven't been there they don't know. I never thought I was going to become an addict growing up, you know? It happened. See, when I went in my first doctor's appointment with my son I decided I was going to do everything the legal way and try to get on the Suboxone program, because I knew about it prior to that, just try to have a healthy pregnancy. So when I went in and I talked to my OB at first I explained to her, "I am addicted to opiates and I don't want to use while I'm pregnant, and my other kids were taken," and whatever. So I confided in her that I've done Suboxone off the streets before...right off the bat she just looked at me different and just made the whole situation uncomfortable for me. So my second visit when I went back, I just told her, "I kicked the habit on my own," whatever, when really I just went and bought a bunch of Subutex off the street. (Former MOUD client)

Real and perceived received stigma from medical and social service providers can also greatly limit women's interest in disclosing or seeking treatment for OUD.

I think women do not feel like they will have any kind of a respectful experience if they acknowledge both substance use, abuse, and pregnancy. There's so much judgment that comes into the picture, and for women especially. We know that they don't always have the support. Men tend to have women in their lives to take care of the children if they go to jail, if they go to treatment. Women generally don't have that kind of experience. (Helena nonprofit) 
[There's] definitely a level of fear if you're a mother. A level of fear of you don't want to get CPS involved in your case at all. And if you admit that you have this problem and you go seek help for this problem, you're automatically going to be on their radar, I think is a big fear for people reaching out for help. (Polson maternal health provider)

Received stigma, both real and perceived, from medical and social service providers toward pregnant women undermines the trust necessary to engage women with OUD in both prenatal and postpartum care and OUD treatment. Some providers recognize the lack of trust and compensate for it by avoiding conversations and screening for OUD to ensure that women continue to seek prenatal care.

A lot of times we kind of lean towards not doing drug screening, drug testing, just because, if they can get some kind of prenatal care, even though you know they're using or whatever, you can at least educate them and stuff when they come in for their visits, and they don't just disappear to where you never see them again. (Helena maternal provider)

I think there was talk of doing some universal urine screens to kind of identify women, but I think it's just hard when we work in an area where people have... They do have some choices in $\mathrm{OB}$ care, and so if you have that reputation at a clinic where one clinic will be testing and one won't, we would see women getting their initial $\mathrm{OB}$ care at one and then transferring to us to delivery, and so it's kind of difficult in that way. (Polson maternal health provider)

Avoiding screening to continue to ensure prenatal care might improve some physical health outcomes for the woman and her fetus. However, the lack of screening in the prenatal period also leads to less support in the postnatal period. When coupled with the distrust outlined above, avoiding the topic of OUD throughout the prenatal and postpartum period is understandable, but may and lead to suboptimal outcomes for women and their children.

And [lack of screening is] not fair either because that mom goes home and if she's got $a$ baby and if she's been using opioids in order to get through her days and cope with her life, she's sent home with a baby who then goes into withdrawals two to three days later and that is terrifying. I can't imagine having to sit there with that knowledge that you've done your best to hide this in order to keep your kids, but then you have to and you are watching one of them be in pain. So there's this piece of we don't screen well and then we don't have a solid system of if I answer yes to this question, what happens to me? The mistrust that's still really there in a lot of those provider conversations is totally understandable. I really don't fault people for this in those conversations. I don't know what I would do. I've never sat in that seat and I hope I don't because those are some pretty tough choices. (Helena nonprofit)

Stigma from providers contributes to the lack of integration across the prenatal and postnatal period, as well as across prenatal care and OUD treatment and recovery. In addition, stigma from the broader community, friends, and family can limit a woman's ability to seek treatment.

They just need to get their quote, unquote, "Shit together." They just need to make better life choices. I'm not sure if the majority of the community sees it as a disease. I think those of us in the field are understanding. I think those of us that work in the trenches can understand and kind of experience firsthand the disease. But I think our overall community and even Montana culture has a big 
stigma on, if you're an addict, you must be the one to blame, right. Unworthy. Whatever word you want to throw in there. We have a lot of work to do. (Polson behavioral health provider)

I think the other biggest issue is supportive family and significant others in seeking out MAT, MAT services. It's kind of the, we still definitely have the community attitude of you don't need that. That's just a crutch, you know, you can quit on your own, all that kind of stuff. So I would say that's a challenge. (Polson behavioral health provider)

This received stigma from the community can reinforce the self-stigma that many pregnant women with OUD and SUD feel, especially in the context of traditional ideals of motherhood.

So this is kind of my own observation here, is guilt. A lot of guilt. And a lot of self-shame, self-blame. When I start working with mama's even post-delivery, there's so much guilt. And I think when you look at motherhood alone, it's hard not to kind of move into that role without a lot of second guessing and a lot of questioning, a lot of societal pressures on what it's supposed to be versus kind of what it really is. So I could imagine that moms who are pregnant, who are also addicted, could have a lot of that self-blame, shame, guilt..." (Helena behavioral health provider)

The stigma of actually being pregnant, people knowing you're pregnant, knowing that you're also struggling, living within a rural setting where secrets are not a real thing and then also even untruths are really persistent around [MOUD]. (Helena nonprofit)

Some providers talk about the potential for positive change that pregnancy and OUD can create, especially since MOUD is a feasible treatment option while pregnant. However, the motivation created by pregnancy is usually not enough to enable long-term recovery in the absence of consistent medical and behavioral health care, as well as social support.

Pregnancy can certainly lend to motivation for change, but this is not something typically that people can do alone, to be able to say "Well I'm just going to stop." People usually need a lot of support in order to do that, but then you have the pregnancy and you think well, if I admit to this now, then what? (Helena behavioral health provider)

I think there are a lot of women who have positive stories about how their pregnancy really brought them to a place to consult with or deal with or confront the substance abuse disorder. There's also a lot of women who haven't, that didn't do it. I can engage in conversation with them after that and they're like, "If being pregnant wasn't enough for me to stop, what is?" And so there's a little bit of a double edged sword, I feel like. I couldn't do that. The shame and guilt. All parents, that force of parent guilt is not to be underestimated, but working with pregnant women and watching them struggle with substances, that is dialed up to a level that is just so unfathomable for me. (Polson maternal health provider)

I really think a lot of this is driven by poverty, diseases of despair, the inability to access care when you need it and watching families just constantly live on the edge. The amount of stress and anxiety, understanding that they're just trying to cope and this is what they're trying to get by with. Thinking about that mom that did abstain throughout her entire pregnancy and she has made it to 
that six week postpartum and she's got a crying baby and she's overwhelmed and doesn't have a job and doesn't know where they're going to live. Just understanding in that moment, I can understand how you'd want to get lost. (Helena behavioral health provider)

\section{Gaps in the OUD treatment system for pregnant women}

\section{Provider knowledge and training}

Because this study focused on the perspectives and experiences of providers who work with pregnant women with OUD, their level of knowledge about both prenatal options for OUD treatment, as well as ongoing OUD treatment and recovery support in the postpartum period, was quite high. However, as noted when discussing stigma, many providers described a lack of knowledge on the part of their peers and colleagues in regard to how to support pregnant women with OUD, especially those seeking MOUD.

I think it would be education for both community and then higher-level providers on what trauma sensitive, gender specific care looks like, that medical care for women especially because of some of the things I talked about before. They tend to be the sole caregivers of their children. They don't have a lot of support to fall back on. They want to keep their children with them. They don't want to lose their kids. They can't ... If they go to jail, their whole life is turned upside down. They lose their jobs. They lose their children. They don't have anything to fall back on.... think the idea that a lot of women have experienced trauma and are re-traumatized when they go through the system, whether it be for medical, mental health care, when they have to see countless different providers, tell their story over and over and over again. That's extremely detrimental. I think I would love to see community-wide, including providers, education about gender specific care and also trauma sensitive care. (Helena nonprofit)

And I guess kind of delve into the different patient populations and what those patient populations may look like within their treatment plan too for the medical providers would be most beneficial, I would think, as an extension of just the general MAT waiver program. (Helena behavioral health provider)

Some providers highlighted the need for more information and training on both local resources and best practices in treating pregnant women with OUD.

And St. Pete's [the local hospital] has a vast amount of services, but I couldn't tell you everything that St. Pete's offered. They're our health center here in town and they're the only hospital shop within so many miles around, but I couldn't tell you all the services that they provide unless they broke it down into a specialty and they promoted, I don't know, OB services or orthopedics or gastroenterology, I don't know what it might be, or, "We got a new surgeon," that kind of thing. Unless you have that promoted or put in your face, you really don't know that that service is offered. (Helena behavioral health provider)

I do think the cost of some of these programs is prohibitive. It would be nice to be able to access trainers of trainers in Montana for various programs at no charge. This cost issue is a real barrier for some of that. Mindset is one thing, but cost is another. And if we had people who routinely would come to the state and do training of trainers for various programs, it would increase our capacity 
to be able to train when people leave the field. We do have a high burnout rate.

(Helena behavioral health provider)

\section{Ongoing treatment and recovery services}

Many providers emphasized the importance of the postpartum period in initiating or maintaining recovery and treatment. As noted in the billing section, state support for ongoing care authorizations for postpartum women is a critical variable that increases the likelihood of long-term recovery.

We often find about the six-week mark, and this is pregnant women or postpartum women in general, there tends to be a huge hormonal shift. And so a person who's experienced a large amount of trauma, who has co-occurring disorders, and who's got the card stacked against them at that six-week mark, the postpartum issues really are exacerbated. So if we can get them past this mark, at least we can work through the mental health issues. The hormonal changes that come up and oftentimes craving to use drugs because throughout the pregnancy, there's a little bit more motivation to not use...but if we can keep them there for three months after we can work through med management and any changes with that process, anything that they need to process in their mental health groups and individuals and with their LAC, the outcomes are much better. (Helena behavioral health provider)

In the postpartum period, women with OUD who wish to continue receiving or initiate treatment have an extremely difficult time finding treatment situations that fit for them. This is especially true for inpatient treatment and recovery residence options that allow babies and young children. Providers consistently highlighted the need for treatment options that support women with children in a way that allows them to stay in their communities and with their children, a practice that has been shown to improve outcomes for women and their families.

That's the thing that's been missing. Everybody has to go away to get treatment. I like drug court because it teaches you how to be sober in your community and reintegrates you back into the community when it comes to the criminal aspect. But I think that there needs to be a treatment center, even a treatment center for pregnant women, sober living houses. The lack of housing is ridiculous around here. (Polson social service provider)

There are the only three places that I'm aware of in the state that take Medicaid that are able to take mothers and children, but both of them have limits on the number of children that can be there. And there are no facilities for men and children, fathers and children. There just aren't. Like having a facility where a family could move in or your whole family, mom and dad and kids could move in and be someplace safe and get treatment would be great. We don't have that. (Helena social service provider)

In addition to the need for specific treatment and recovery residence options that are childfriendly, many providers also highlighted the need for integrated care in the postpartum period for women with OUD and their families. This could be supportive housing, or wraparound services that integrate medical care, social supports, and housing. Key to ongoing treatment and recovery support is making sure women can maximize their personal support networks and that they can remove themselves from unsupportive family and social situations when necessary. 
I think that [we need] one more stair-step into having some level between complete community-based outpatient [treatment] and a residential program. Really having a layer built in there where there is support wrapped around a mom and her child almost 24/7, maybe not legitimately 24/7, but really the idea of co-located services being able to live in a really supportive environment. Because so often there are so many other challenges that are coming along with that drug use, that substance use, for a parent and especially a mom, that she may not have the family support or those community support and building a system around them. (Helena maternal health provider)

The struggle that we ran into time and time again, and we, I think, as a group, at least in Wrapped in Hope, identified as our biggest barrier was that oftentimes these women were continuing to live in homes that everyone was using. So, their child would be removed and be placed with their sister, but their sister was also using, and there was no drug screen from the sister, and so the child could remain in the home with the mom, which was great, especially when Mom was sober, but all the family members have continued to use, and that was often the trigger, often a factor related to relapse. We would get asked all the time, "Can you just help me find a place to go, to move out? We need this. We need some assistance with rent," and that was just something we just didn't have the ability to provide all the time. So, that was a huge barrier. (Polson maternal health provider)

In a big picture sense, many providers highlighted the need to integrate across medical and behavioral health care, across the prenatal and postnatal period, and across all kinds of physical and social needs to support women, especially those who are pregnant and with young children. More complete system integration, and the resources to maintain it, would not only decrease the gaps in care from a capacity standpoint, but could also address some of the barriers to treatment created by stigma and a lack of trust in the system, because investment would suggest support.

I think I would have a system of care that embraced women, and this is hard to even imagine what that would look like, would be non-judgmental, where someone could walk into a clinic whether in crisis or not. But a) we need something for women in crisis because we don't have that. And they would be able to access services very quickly. There would be no waiting. There would be identified professionals that would have time in their schedule set aside that would be able to see someone within 24 hours of walking into the system and saying, "I'm in, I need help. And I need it now."...Right, right. That we're going to embrace whatever we can and help you to help yourself and not to demonize you. 'Cause I think women, regardless of whether it's perinatal mood and anxiety disorders or substance use disorders or co-occurring, I think there's still a tendency of feeling stigmatized and demonized, like they're somehow very bad people. (Helena maternal health provider)

\section{Success stories}

Success stories feature both positive outcomes for individual women and characteristics of effective treatment and recovery systems for pregnant and postpartum women with OUD. Two stories here highlight the positive impact of integrating across prenatal and postnatal care, and across the medical and behavioral health care systems.

For example, last year we [had] a woman that was with us for at least a year. She had four kids who were basically age two through ... The oldest one I think 
was 10 , and then she was pregnant with us, as well. She was able to be with us the entire time. She found out she was pregnant, was housed with us, and then had her baby with us, and then moved into permanent housing through the Helena Housing Authority. We are still following-up with us, especially with case management, and she's doing very, very well. (Helena nonprofit)

There's an awesome article about St. James and also the Montana Chemical Dependency Center out of Butte. Their relationship, they came together. They weren't working together before, but they developed a relationship, were able to extend the gestation period at which they would treat women at MCDC. They used to turn them home, quote, unquote, to give birth because they couldn't provide that level of medical care safely. Well, now they're partnering with the hospital that's five minutes away from them and are able to do so without a transition. (Helena maternal health provider)

Ultimately, using success stories as a means of outreach and raising awareness may decrease the stigma and lack of information associated with pregnancy, parenting, and OUD.

I think maybe more than telling the positive stories, it's telling the story of this happens because we don't talk about it and I think a lot of families whether they're in recovery phase or still in active use phase or whatever, there's a lot of hurt and shame about that. I think just letting both women and also the families that are supporting them know that they're not alone and that there is treatment. I think that's the message that I think we need more than we need a feel-good story. (Helena nonprofit) 


\section{IMPLICATIONS AND RECOMMENDATIONS}

The results of this study underscore some of the observations made at the national level about the challenges for pregnant women with OUD in accessing treatment, as well as lessons learned in related studies within the state of Montana, focused on barriers to OUD treatment and challenges faced by pregnant and postpartum women with SUD. In addition, these results specific to pregnant women with OUD in Montana add nuance to understanding the challenges and potential opportunities for improve access to care and outcomes.

Screening pregnant women for opioid use is not universal in Montana: The MT PRAMS data suggests that $18 \%$ of pregnant women are not asked about illicit drug use during prenatal visits, and an additional $12 \%$ do not receive prenatal care in the first trimester. This means that almost one-third of pregnant women in Montana are not directly screened for OUD, nor are they likely to receive information about MOUD or other treatment options. Interviews with providers corroborate these numbers, with many providers noting that they choose not to screen some patients for fear that the patients will not come back for subsequent prenatal care if pressed about their substance use.

Recommendation: Improve universal screening rates for illicit substances in prenatal care and expand integrated behavioral health (IBH) approaches like the Meadowlark Initiative, including MOUD, within prenatal care settings so that pregnant women with OUD can receive supportive, comprehensive care in one location.

Integrating prenatal care and MOUD, as well linking care from prenatal to postpartum periods, is seen by providers to have a positive impact on outcomes: In the two locations profiled in this report, both have some integration of prenatal care and MOUD. In Polson, an MOUD program has been integrated into a hospital system's prenatal care for several years, and linkages continue into the postpartum period. Providers stated the positive impacts for pregnant and postpartum women regarding continuity of care. In Helena, integration of MOUD and prenatal care in the hospital system is new. Providers noted the prior challenges for pregnant women, such as having to go to separate clinics and medical systems to receive each type of care separately. In both communities, there are specific programs and organizations that focus on OUD treatment in the postnatal period, but these are usually not directly connected to the medical systems providing prenatal care.

Recommendation: Expand IBH in prenatal settings and invest in coordinated care systems that continue into the postpartum period. Conversely, expand basic prenatal care in primary care settings that also offer MOUD to provide a single location for all types of care needed by pregnant women with OUD.

Stigma from providers and from themselves creates barriers to pregnant women with OUD seeking treatment and prenatal care: Stigma from some medical providers about OUD and pregnancy can lead to judgement, critique, and 'divergent' care for pregnant women. Past experiences of stigma received from medical providers about SUD can lead pregnant women to avoid prenatal care out of fear that they will be judged. Self-stigma can generate additional shame and fear for the fetus for women with OUD who learn they are pregnant and feel that they are bad parents. Both types of stigma can push pregnant women with OUD further from receiving any type of treatment or prenatal care.

Recommendation: Reduce stigma among maternal health providers through training about MOUD and engagement with existing MOUD providers. Reduce stigma among pregnant women through education and outreach campaigns that highlight the opportunities for treatment rather possible punishment for substance use. 
Increased punishment and lack of treatment capacity for women with OUD who have children creates barriers to initiating treatment and sustaining recovery: Many providers underscored the extreme fear that pregnant women have that admitting any type of SUD will lead to their children being taken away. There are few treatment and recovery options within the state that allow women to bring their children with them, so seeking treatment means leaving one's children. For women in the postpartum period, leaving their newborn to seek treatment may have potentially negative impacts on both the mother and the child.

Recommendation: Expand treatment and recovery options that are accessible to women with babies and young children. Increase funding for recovery residences that support pregnant and postpartum women, as there is a specific lack of recovery residences in the state of Montana that allow children to reside with their mothers. Incentivize quick engagement with the treatment system by women whose children are temporarily removed and promote reunification once SUD recovery is stable. 


\section{CONTACT INFORMATION}

\section{Kristal Jones}

kristal@jgresearch.org

Brandn Green

brandn@jgresearch.org 